\title{
Single-domain h-BN on Pt(110): Electronic structure, correlation, and bonding
}

\author{
Marco Thaler $\odot,{ }^{1}$ Dominik Steiner, ${ }^{1}$ Alexander Menzel, ${ }^{1}$ Florian Mittendorfer $\odot,{ }^{2}$ and Erminald Bertel $\odot{ }^{1, *}$ \\ ${ }^{1}$ Institute of Physical Chemistry, University of Innsbruck, 6020 Innsbruck, Austria \\ ${ }^{2}$ Institute of Applied Physics and Center for Computational Materials Science, University of Technology, 1040 Vienna, Austria
}

(Received 19 June 2020; revised 1 September 2020; accepted 12 October 2020; published 29 October 2020)

\begin{abstract}
Extended single-domain growth of h-BN is observed on $\mathrm{Pt}(110)$, if the precursor molecules are deposited at sufficiently high temperatures. We examined the electronic structure of the h-BN/Pt(110) system by angle-resolved photoemission (ARPES), work-function measurements, and density-functional theory (DFT) calculations. van der Waals forces dominate the h-BN/Pt(110) interaction by far, although DFT analysis of the local density of states reveals the existence of a local covalent interaction of some $\mathrm{N}$ atoms with $\mathrm{Pt}$ surface atoms. The local bonding contributions cause the appearance of a $(1 \times n)$ missing-row reconstruction $(n=5$ or 6$)$ of the $\mathrm{Pt}$ (110) surface, if the system reverts to room temperature after h-BN adlayer formation at $1120 \mathrm{~K}$. This unique phenomenon of the template adapting to the adlayer structure mitigates differences in the thermal-expansion coefficient upon cooling. The h-BN $\pi$ bands hybridize with Pt $d$ bands. Nevertheless, the dispersion of $\pi$ and $\sigma$ bands as measured by ARPES is overall well represented by the free-standing monolayer band structure except for the appearance of replica bands induced by the Moiré structure. A comparison between the experimentally measured $\pi$ bands and the band structure obtained from DFT slab calculations suggest the existence of significant correlation effects in photoemission from h-BN/Pt(110). The locally varying distribution of N-Pt hybrid states straddling the Fermi level indicates a corresponding spatial variation of the chemical reactivity.
\end{abstract}

DOI: 10.1103/PhysRevResearch.2.043156

\section{INTRODUCTION}

Monolayers of hexagonal boron nitride (h-BN) on various substrates have been investigated vigorously in recent years [1]. The interest was mainly driven by the prospect of using $\mathrm{h}-\mathrm{BN}$ as insulating layer in graphene-based electronic devices, thus constructing a base for two-dimensional (2D) electronics or even spintronics. However, other attractive features of hBN emerged, such as formation of a structured template for cluster deposition [2-4], catalytic activity [5,6], or hosting single-photon sources with unprecedented spectral properties $[7,8]$. Accordingly, a vast literature on growth and properties of h-BN on a multitude of transition-metal surfaces has accumulated and was recently summarized in a comprehensive review by Auwärter [1]. The electronic structure of h-BN monolayers has been examined mostly on graphite, graphene, and metal substrates with hexagonal symmetry [9-15]. Very recently, Achilli et al. [16] and Steiner et al. [17] added another template for h-BN monolayer growth, namely $\operatorname{Pt}(110)$, to the extensive database. Surprisingly, $\operatorname{Pt}(110)$ proved to be an excellent substrate for large-scale, single-domain growth of h-BN. Obviously this cannot be understood in the usual scheme of epitaxial growth placing emphasis on lattice

\footnotetext{
*erminald.bertel@uibk.ac.at
}

Published by the American Physical Society under the terms of the Creative Commons Attribution 4.0 International license. Further distribution of this work must maintain attribution to the author(s) and the published article's title, journal citation, and DOI. (mis)match, rather it was attributed by Steiner et al. to the high mobility of $\mathrm{Pt}$ surface atoms at the appropriate growth temperature [17].

This conclusion matches with the observations of Lee et al. who showed liquid gold to be a nearly ideal substrate as well [18]. While the growth of $\mathrm{h}-\mathrm{BN}$ on $\mathrm{Pt}(110)$ is best carried out at $T>1120 \mathrm{~K}$, when the Pt surface atoms form a quasiliquid layer, the $\operatorname{Pt}(110)$ surface reverts to $(1 \times n)$ missing-row (m.r.) reconstruction upon cooling to room temperature, $n$ being 5 or 6 . This is an extraordinary case, where the substrate adapts its structure to the overlayer instead of the other way around. The single-domain growth on a quasiliquid layer argues for a weak, almost pure van der Waals interaction, while the restructuring of the $\operatorname{Pt}(110)$ surface upon cooling indicates a more specific bonding interaction. This motivated us to carry out a detailed investigation of the electronic structure and bonding of h-BN on $\mathrm{Pt}(110)$.

\section{EXPERIMENT AND THEORY}

For angle-resolved photoemission spectroscopy (ARPES) the sample was mounted on a two-axis goniometer connected to a liquid-nitrogen reservoir. All ARPES measurements were carried out at room temperature. The photoelectrons were detected with an Omicron AR 65 hemispherical analyzer which, too, was mounted on a two-axis goniometer. An energy resolution of $100 \mathrm{meV}$ was used for all ARPES spectra. Workfunction measurements were carried out with a sample bias of $-10 \mathrm{~V}$ in order to reliably detect the low-energy cutoff of the secondary electron peak [19]. For the preparation of the h-BN monolayer, Borazine was dosed through a capillary at a 


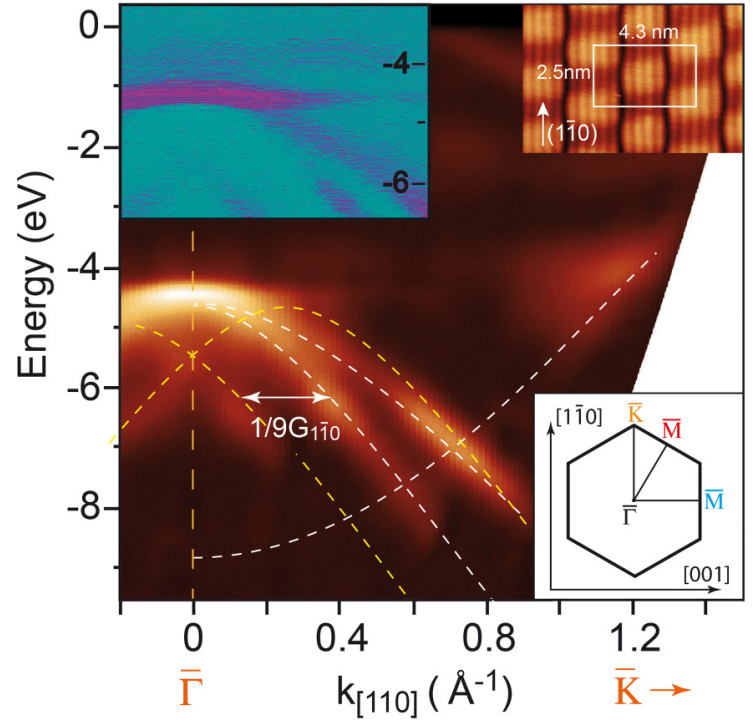

FIG. 1. Band dispersion of h-BN/Pt(110) along $\bar{\Gamma} \overline{\mathrm{K}}$ measured with He I. Dashed white dispersion curves are from Blasé et al. [28]. Dashed yellow curves are Moiré induced replica bands. Upper left inset: Gradient enhanced map of the $\sigma$ band around $\bar{\Gamma}$ showing a flat-band tail; upper right inset: STM image of h-BN/Pt(110) showing the Moiré pattern with the white rectangle delineating the centred rectangular unit cell. Lower inset: 2D Brillouin zone of h$\mathrm{BN}$; the rectangular coordinates denote the high-symmetry directions of $\operatorname{Pt}(110)$.

sample temperature of $\sim 1170 \mathrm{~K}$. The procedure was the same as the one used in our previous scanning tunneling microscope (STM) examination of the h-BN/Pt(110) system [17]. A corresponding low-energy electron diffraction (LEED) pattern is shown in the Supplemental Material [19]. The borazine gas reservoir was at room temperature, but the gas pressure in the reservoir was limited to below 200 mbar in order to keep the polymerization rate at a tolerably low level. For the presentation, the ARPES intensity distribution maps were processed using the GWYDDION image processing software [20]. From the raw data we subtracted parabolic backgrounds, for the inset in Fig. 1 we used the gradient detection algorithm with a vertical Sobel kernel, and for Fig. 2(b) the contrastenhancement algorithm was used.

The density-functional theory (DFT) calculations were performed using the Vienna Ab-initio Simulations Package (VASP) $[21,22]$ with the Perdew-Burke-Ernzerhof (PBE) xc functional [23] and DFT-D3 corrections [24] to include dispersion interactions. Brillouin-zone integration was carried out on a $2 \times 2 \times 1 k$-point mesh. The substrate was represented by a six-layer $\mathrm{Pt}(110)$ slab with an optimized DFT-D3 lattice constant of $3.92 \AA$. For the h-BN layer a $(10 \times 10)$ superstructure was assumed. Relaxation of the BN adlayer and the four uppermost substrate layers was performed until the residual forces were smaller than $0.01 \mathrm{eV} / \AA$. One has to be aware that the actucal choice of the van der Waals (vdW) correction scheme can have a pronounced influence on the adsorption energy [25], However, the comparison of the calculated structure with the experimental data, in particular the excellent agreement between the Tersoff-Hamann simulation

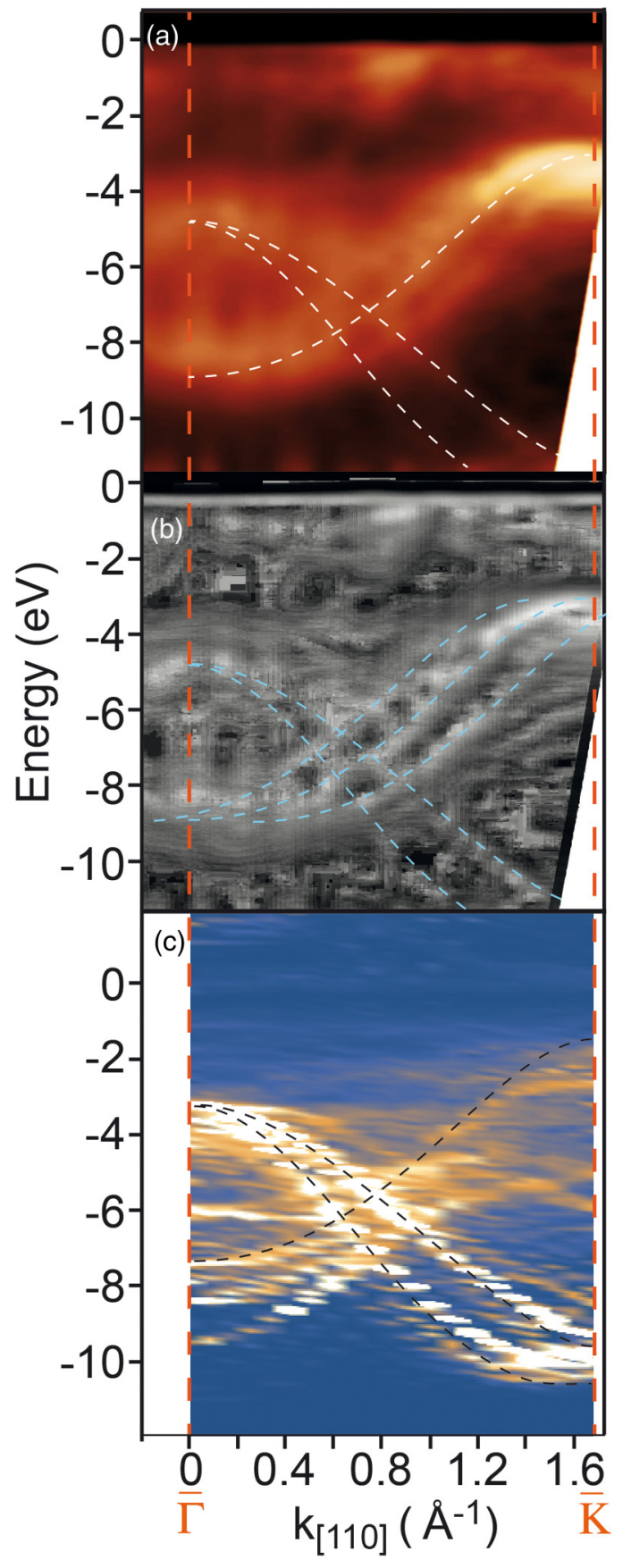

FIG. 2. Band dispersion of h-BN/Pt(110) along $\bar{\Gamma} \overline{\mathrm{K}}$ measured with He II. Dashed dispersion curves are from Blasé et al. [28]. (a) Original data. (b) Image processed with local contrast enhancement; here, the dashed lines include the replicas of the $\pi$ band. (c) DFT band structure calculation with bands unfolded as described in the text. The intensity reflects the magnitude of the projection onto the h-BN layer. The dashed lines are copied from Fig. 1 and aligned with the $\sigma$ bands at $\bar{\Gamma}$.

and the experimental STM data [17], lends confidence to the present results.

To retrieve the $\mathrm{h}-\mathrm{BN} / \mathrm{Pt}(110)$ primitive cell band structure we used an unfolding technique implemented in the BANDUP code by Medeiros et al. [26]. In addition, we projected the 
resulting bands onto the h-BN layer and represented it as a spectral weight function [27].

\section{RESULTS}

\section{A. Angle-resolved UV photemission \\ 1. He-I data: band dispersion}

Figure 1 shows a comparison of our ARPES measurements along the $\bar{\Gamma} \overline{\mathrm{K}}$ direction with theoretical band calculations. The dashed white lines are $G W$ calculations for the freestanding monolayer by Blasé et al. [28]. The $\pi$ band is hardly observed in the He-I data. The $G W$ calculation captures the dispersion of the bands slightly better than a grid-based projected-augmented wave density functional theory (GPAWDFT) calculation [29].

There are two additional bands, crossing each other at $\bar{\Gamma}$. These bands do not arise from the Pt substrate, but they can be interpreted as backfolded bands as indicated in Fig. 1: They have the same slope as the $\sigma$ bands and are displaced along the $k$ axis by $1 / 9 G_{11}$, with 9 being the closest commensurate approximation to the Moiré periodicity in the [11̄0] direction and $G_{1 \overline{1} 0}$ the reciprocal-lattice vector of the clean Pt surface $[16,17]$. Furthermore, a flat-band feature extends from the top of the $\sigma$ bands at $\bar{\Gamma}$ towards $\bar{K}[30,31]$ (upper left inset of Fig. 1).

The top of the $\sigma$ bands is found at $4.6 \mathrm{eV}$ below $E_{\mathrm{F}}$, whereas it is located at $3.1 \mathrm{eV}$ in the DFT calculation shown in Fig. 2(c). A few weak structures between the h-BN related bands and the Fermi level are primarily due to Pt bands (compare Fig. 5 in Minca et al. [32]). Dispersion plots in the $\bar{\Gamma} \bar{M}$ and $\bar{\Gamma} \overline{\mathrm{M}}^{\prime}$ directions are shown in the Supplemental Material [19].

\section{He-II data: Band dispersion}

The band dispersion in the $\bar{\Gamma} \overline{\mathrm{K}}$ direction as measured with $\mathrm{He}$ II is displayed in Fig. 2. Again, the experimental dispersion is quite well reproduced by the $G W$ calculation of Blasé et al. [28]. In our measurements the $\pi$ band appears as a rather diffuse structure in contrast to the He-II measurements reported by Achilli et al. [16]. Paradoxically, this is due to the better film quality in the present case, where the h-BN has been grown at $T>1120 \mathrm{~K}$ [17]. Here, the $\mathrm{Pt}(110)$ surface exhibits much larger terraces and the h-BN monolayer is almost free from defects and domain boundaries. Consequently, the periodicity of the Moire pattern is very regular and gives rise to replica bands, as they are also observed for the $\sigma$ bands in the He-I data. Thus, the intensity of the $\pi$ band is distributed over the "ghost bands," which renders it less distinct as compared to the more perturbed h-BN films obtained from growth below $1100 \mathrm{~K}$. The triple structure of the original and backfolded $\pi$ bands of a free-standing monolayer is quite clearly seen in the contrast enhanced image of Fig. 2(c). Since the umklapp displaces the replica bands horizontally, the binding energies at $\bar{\Gamma}$ and $\bar{K}$ remain well defined and are 9 and $3.2 \mathrm{eV}$, respectively. In contrast, the DFT calculation shown in Fig. 2(c) indicates a pronounced hybridization of the $\pi$ bands with the Pt substrate, giving rise to a broad manifold of bands, spanning an energy range of 3-4 eV throughout the PCBZ. The centroid of the DFT $\pi$ bands ranges from $8 \mathrm{eV}$ at $\bar{\Gamma}$ to 4

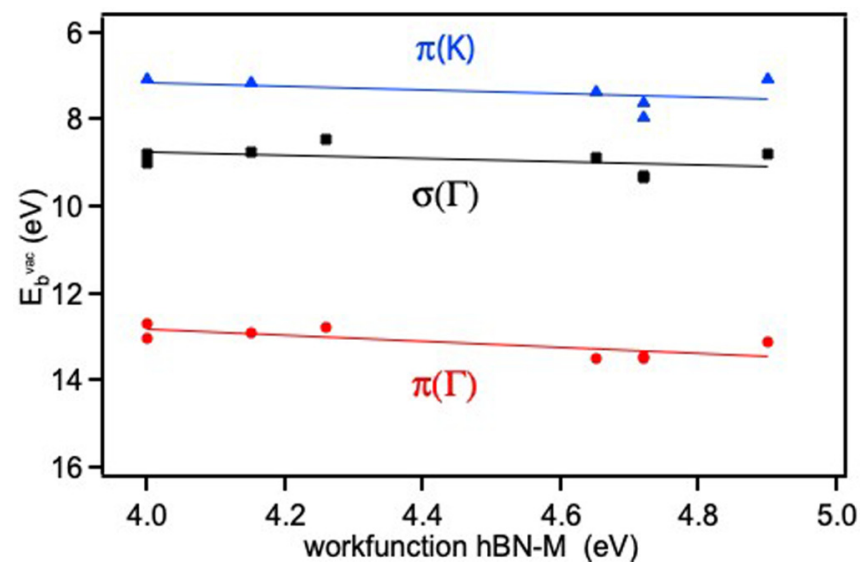

FIG. 3. Binding energies of h-BN $\pi$ and $\sigma$ bands referred to the vacuum level for different h-BN metal systems listed in Table I. Lines are best fits to the data points. For $\mathrm{Rh}(111)$ and $\mathrm{Ru}(0001)$ only $\sigma^{\alpha}$ and $\pi^{\alpha}$ energies are included. The data for $\mathrm{Ni}(111)$ are omitted, since this is a strongly interacting system. They would fall somewhat below the trend lines.

$\mathrm{eV}$ at $\bar{K}$. Thus, the average binding-energy difference between DFT and experiment seems to be smaller than for the $\sigma$ bands.

\section{B. h-BN-metal bond: Work-function and DFT results}

In ARPES, the top of the $\pi$ band lies $3.2 \mathrm{eV}$ below $E_{\mathrm{F}}$, in the DFT calculation at only slightly lower binding energy. This places the Fermi level approximately in the middle of the band gap. In order to obtain the binding energy with respect to the vacuum level $E_{\text {vac }}^{\text {bind }}$ we determined the work function $\Phi$ from the total width of the photoelectron spectrum. See the Supplemental Material for technical details [19]. For the pristine Pt surface we obtained $\Phi=5.48 \mathrm{eV}$, close to the literature value [33]. The work function of the $h-B N /(1 \times n)$ m.r. $-\operatorname{Pt}(110)$ surface is $760 \mathrm{meV}$ lower, i.e., $\Phi_{\mathrm{hBN}}=4.72 \mathrm{eV}$. Table I shows a comparison of binding energies for a variety of metals.

In Fig. 3 we compare work-function and binding-energy data for several different h-BN/metal systems. As noted previously [11], the binding energies of $\pi(\overline{\mathrm{K}})$ and $\sigma(\bar{\Gamma})$ with respect to vacuum energy stay more or less constant over a range of systems with work functions differing by $\sim 1 \mathrm{eV}$. This is also true for the newly added data points of $\mathrm{h}-\mathrm{BN} / \mathrm{Pt}(110)$ at $\Phi_{\mathrm{hBN}}=4.72 \mathrm{eV}$.

It may be interesting to compare the present $\pi$-band data with a rather different system, i.e., an h-BN monolayer grown epitaxially on graphite and graphene. The Fermi-level related binding energy was found to be $\pi(\overline{\mathrm{K}})=2.8 \mathrm{eV}$ for $\mathrm{h}$ $\mathrm{BN} /$ highly oriented pyrolytic graphite [9], and $\pi(\overline{\mathrm{K}})=3.5 \mathrm{eV}$ for $\mathrm{G} / \mathrm{SiC}[10]$. In the latter case the bandwidth was found to be significantly reduced: $\pi(\bar{\Gamma})=7.9 \mathrm{eV}$. Koch et al. [10] enumerate strain, island morphology, and azimuthal disorder as possible causes of the anomalously small $\pi$ bandwidth.

The DFT(PBE-D3) calculations predict an average adsorption energy of only $E_{\mathrm{ads}}=-0.244 \mathrm{eV}$ per formula unit (f.u.) $\mathrm{BN}$. Turning off the vdW corrections reduces the energy even further by an order of magnitude $\left(E_{\mathrm{ad}} \sim-0.030 \mathrm{eV} /\right.$ f.u. $)$, indicating that the adsorption of the major part of the $\mathrm{BN}$ sheet is driven by van der Waals-like forces. 
TABLE I. Work-function changes and binding energies for h-BN on various transition-metal surfaces. $E_{\text {bind }}^{\text {vac }}$ for Pt(110) from Ref. [9] has been calculated by using the present work-function data. For $\mathrm{Rh}(111)$ and $\mathrm{Ru}(0001)$ both values for the split bands are given.

\begin{tabular}{|c|c|c|c|c|c|c|c|c|}
\hline \multirow[b]{2}{*}{ Substrate } & \multirow[b]{2}{*}{$\Phi_{\text {clean }}$} & \multirow[b]{2}{*}{$-\Delta \Phi$} & \multicolumn{2}{|c|}{$\pi(\bar{\Gamma})$} & \multicolumn{2}{|c|}{$\sigma(\bar{\Gamma})$} & \multicolumn{2}{|c|}{$\pi(\overline{\mathrm{K}})$} \\
\hline & & & $E_{\text {bind }}^{F}$ & $E_{\text {bind }}^{\mathrm{vac}}$ & $E_{\text {bind }}^{F}$ & $E_{\mathrm{bind}}^{\mathrm{vac}}$ & $E_{\text {bind }}^{F}$ & $E_{\text {bind }}^{\mathrm{vac}}$ \\
\hline $\mathrm{Ni}(111)[11]$ & 5.30 & 1.70 & 10.30 & 13.90 & 5.30 & 8.90 & 4.57 & 8.17 \\
\hline $\operatorname{Pd}(111)[11]$ & 5.30 & 1.30 & 8.70 & 12.70 & 4.80 & 8.80 & 3.08 & 7.08 \\
\hline $\operatorname{Pd}(111)[12]$ & 5.65 & 1.39 & 8.50 & 12.76 & 4.20 & 8.46 & & \\
\hline $\operatorname{Pt}(111)[11]$ & 5.80 & 0.90 & 8.20 & 13.10 & 3.90 & 8.80 & 2.18 & 7.08 \\
\hline $\operatorname{Pt}(110)[16]$ & & & 8.70 & 13.42 & 4.60 & 9.32 & 2.90 & 7.62 \\
\hline $\mathrm{Pt}(110)$ (present) & 5.48 & 0.76 & 8.78 & 13.50 & 4.55 & 9.27 & 3.2 & 7.92 \\
\hline $\operatorname{Rh}(111)[13,14]$ & 5.50 & 1.35 & $8.76 / 9.83$ & $12.91 / 13.98$ & $4.57 / 5.70$ & $8.72 / 9.85$ & 3.00 & 7.15 \\
\hline $\operatorname{Ru}(0001)[13]$ & 5.44 & 1.44 & $9.00 / 9.83$ & $13.00 / 13.83$ & $4.97 / 5.91$ & $8.97 / 9.91$ & & \\
\hline $\operatorname{Ir}(111)[15]$ & 5.80 & 1.15 & 8.50 & 13.15 & 4.20 & 8.85 & 2.70 & 7.35 \\
\hline
\end{tabular}

Additional information can be obtained from an analysis of the local density of states (LDOS), as provided in Fig. 4. It compares the LDOS for $\mathrm{N}$ atoms (red trace) and $\mathrm{B}$ atoms (blue trace), which are positioned above a missing $\mathrm{Pt}$ row [Fig. 4(a)] or in close contact with a Pt atom [Fig. 4(b)]. For both the $\mathrm{N}$ and the $\mathrm{B}$ atoms above the missing row, the LDOS is very low within the band gap (note that the band gap is underestimated in DFT). In contrast, for the $\mathrm{N}$ atoms (red trace) above the Pt close-packed row, the LDOS within the band gap is considerably enhanced via the hybridization with Pt states and there appears a distinct LDOS peak at and slightly above $E_{\mathrm{F}}$. It can be assigned as a Pt $d-\mathrm{N} p_{\mathrm{z}}$ antibonding feature. The ccupied LDOS below the band gap exhibits the complementary downshift of the bonding parts.

Although the pronounced hybridization might seem to contradict the weak adsorption energy of the BN sheet, a

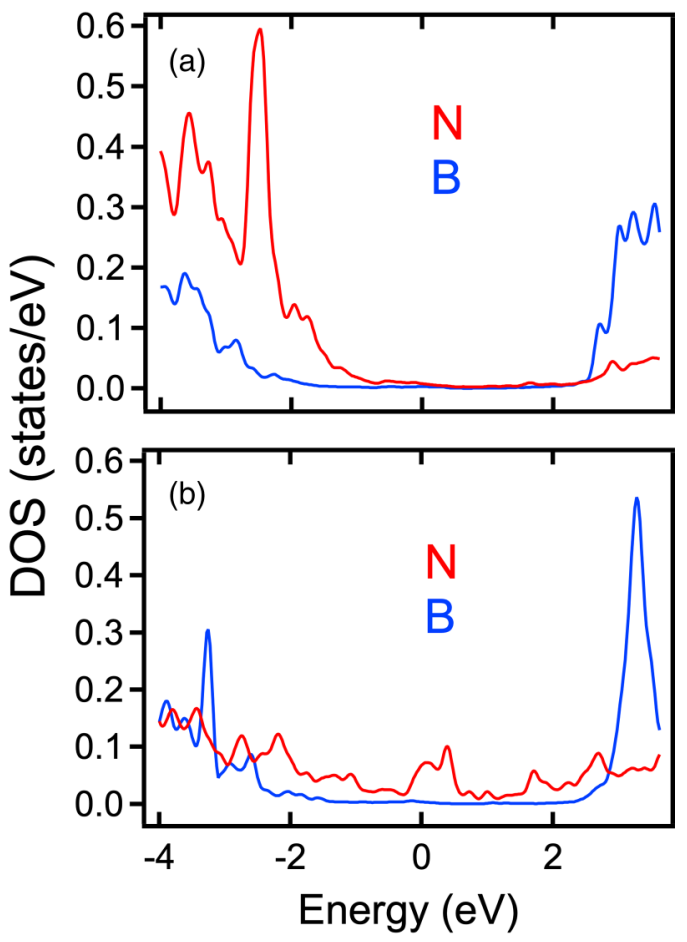

FIG. 4. (a) Comparison of the LDOS for $\mathrm{N}$ atoms (red trace) and $\mathrm{B}$ atoms (green trace) above a missing Pt row (a) and in on-top positions above a Pt close-packed row (b). similar situation was observed, e.g., for the adsorption of graphene/Ni(111) [25] or graphene/Ni/Ir(111) [34].

\section{DISCUSSION}

There are noteworthy differences between DFT theory and experiment. Figure 2(c) shows the results of the DFT slab calculation, where the bands have been unfolded from the $(10 \times 10)$ supercell Brillouin zone $(\mathrm{SCBZ})$ onto the h-BN primitive cell Brillouin zone (PCBZ) [26]. The intensities reflect the magnitude of the projection onto the h-BN layer. This choice is motivated by the small mean free path of the photoelectrons in the He-II spectra.

A first difference concerns the $\sigma$ band binding energy. The $\sigma$ bands are clearly visible in the He-I and rather weak in the He-II data, but even there they emerge clearly in the contrastenhanced image [Fig. 2(b)]. They are also quite faithfully reproduced in the DFT calculation. However, at $\bar{\Gamma}$, the experimental binding energy is about $4.6 \mathrm{eV}$, while DFT yields $E_{\mathrm{b}}=3.1 \mathrm{eV}$. Apart from this overall binding-energy shift of about $1.5 \mathrm{eV}$ the DFT slab calculation, the $G W$ calculation for the free monolayer, and the ARPES $\sigma$ bands agree quite well.

For the $\pi$ bands the binding-energy difference between the experimental spectra and the DFT slab calculation is significantly smaller, although it is not possible to quote a definite figure, since the hybridization with the Pt bands distributes the $\pi$ related states in the DFT calculation over a large energy interval. However, concerning the shape of the $\pi$ bands one notes a better agreement of the ARPES data with the $G W$ free-standing monolayer band structure [dashed lines in Figs. 2(a)-2(c)] than with the present results of a DFT calculation for the h-BN/Pt(110) slab [Fig. 2(c)]. An interpretation in terms of a back-folded monolayer band structure fits the data quite well, while the pattern of the hybrid bands from the DFT calculation exhibits less similarity with the ARPES result. In particular, the splitting of the hybridized $\pi$ bands observed in the DFT results [Fig. 2(c)] is not clearly born out in the ARPES data of Fig. 2(a). In the contrast-enhanced image shown as Fig. 2(b) some structure appears close to $\bar{K}$, which might relate to the hybrid bands obtained by DFT.

Measured binding energies depend of course on the position of the Fermi level, which in real systems can vary depending on the nature and concentration of defect levels within the band gap. However, a defect-induced shift of $E_{\mathrm{F}}$ 
would affect all binding energies in the same way. In contrast, band-specific binding-energy differences could result from final-state effects, because a photohole in the rather localized $\sigma$ bands will be less efficiently screened by the metal substrate than a photohole in the $\pi$ band, which has more overlap with the metal.

The similarity of measured h-BN monolayer band structures with those calculated for free-standing monolayers is a widely observed phenomenon. This is somewhat puzzling in view of the non-negligible hybridization with substrate bands indicated by DFT calculations. A possible cause is correlation in the photoemission final state, i.e., confinement of the photohole within the h-BN layer. Both the differential binding-energy shifts of $\pi$ and $\sigma$ bands with respect to the DFT band structure as well as the virtual absence of h-BN substrate hybridization in the ARPES spectra point to this explanation. In essence this would be a two-dimensional (2D) analog to the paradigmatic 0D example for such an effect in rare earth, namely the observation of atomic $4 f$ multiplets in photoemission, indicating a strong localization of the photohole, whereas in the ground state the $4 f$ levels are hybridized with the $5 d-6 s$ bands [35]. In the rare earth the $4 f$ levels and the $5 d-6 s$ conduction band form a weakly coupled system. The photoemission final state has therefore been described as consisting of a fully screened state, where the $4 f$ photohole is filled by an electron from the conduction band and a poorly screened final state, where the hole remains localized in a $4 f$ orbital and a more diffuse screening charge is built up in the conduction band. Extending the analogy to the present case, one can consider the $\pi$ band of the h-BN monolayer and the Pt conduction band as weakly coupled systems:

$$
H=\sum_{k} \varepsilon_{k}^{P t} c_{k}^{\dagger} c_{k}+\sum_{\kappa} \varepsilon_{\kappa}^{\mathrm{hBN}} a_{\kappa}^{\dagger} a_{\kappa}+\sum_{k \kappa}\left[V(k, \kappa) a_{\kappa}^{\dagger} a_{k}+\text { H.c. }\right] .
$$

Here, $\varepsilon_{k}^{P t}$ is the dispersion of the Pt band (to sketch the idea, it is sufficient to consider only one band) and $\varepsilon_{\kappa}^{\mathrm{hBN}}$ is the dispersion of the h-BN $\pi$ band with $k$ being the crystal momentum in $\mathrm{Pt}$ and $\kappa$ the one in h-BN. Upon photoionization, a hole with momentum $\kappa$ in the $\pi$ band is created, which in turn gives rise to a series of screening and subsequent deexcitation events. The fastest process in this chain of events is the resonant charge transfer from the substrate band into the h-BN monolayer, for which the time scale is set by the matrix element $V_{k \kappa}$. Alternatively, the photohole could be filled via intersystem Auger decay involving metal conduction electrons. However, this process is typically about one order of magnitude slower in van der Waals systems [36]. Specifically, time scales for resonant charge transfer in van der Waals systems are of the order of femtoseconds [37] and for the photoemission process at $h v=40.41 \mathrm{eV}$ about $10^{-17}-10^{-16} \mathrm{~s}$. Thus, photoemission at these conditions probes predominantly the poorly screened final state where the photohole is still confined to the h-BN monolayer. As a consequence, the photoemission spectrum reflects the band structure of the isolated monolayer much like the poorly screened final state in the rare earth, which exhibits the hallmarks of an isolated atom. As an aside, we note that the longer time scale for the photoemission process at lower photon energies may be a reason for shifting the weight to the well- screened final state, which would appear as a more diffuse structure in the spectra. Therefore the virtual absence of the $\pi$ band in the He-I spectra could not only be a consequence of matrix element effects but also be related to the relevant time scales. A general discussion of the interplay between localized and delocalized final states is provided by Fuggle [38].

Next, we address the nature of the replica bands, which are shifted by the reciprocal-lattice vector of the Moiré periodicity in the [110] direction. Interestingly, there seem to exist two different types of "ghost bands" in ARPES spectra of h$\mathrm{BN}$ monolayers on incommensurate substrates. On Ru(0001) [39] and on $\mathrm{Rh}(111)$ [40,41] an energetic band splitting is attributable either to a locally varying interaction strength with the substrate or to differences in local work function. In contrast to this vertical band splitting, a "horizontal" band splitting similar to the present one is reported for h-BN/Ir(111) by Usachov et al. [15]. Due to the absence of a measurable band gap between the backfolded bands and from an analysis of the intensity distribution Usachov et al. concluded that the replica bands are attributable to a photoemission matrix element effect caused by the corrugation rather than an umklapp caused by the periodic potential of the Moiré structure. Our ARPES data, however, do not exclude the existence of a small band gap (see Fig. 1, upper left insert). Furthermore, traces of the replica bands appear in the DFT calculations even after unfolding, i.e., a downwards dispersing intensity displaced by $1 / 9 G_{1 \overline{1} 0}$ towards $\bar{K}$ and the intensity extending more or less dispersionless from the maximum of the $\sigma$ band towards the interior of the PCBZ (see the discussion below). Hence we believe that in the present case the replica bands are conventional potential-induced umklapps.

One should expect the missing rows and the corresponding corrugation of the h-BN layer to produce distinct replica bands in the $\bar{\Gamma} \bar{M}$ [001] direction as well, but the periodicity in this direction is varying between 5 and $6 \mathrm{Pt}$ lattice constants [17]. Accordingly, the scattered intensity contributes to the diffuse background rather than forming well-defined ghost bands [19].

Finally, we discuss the distinct flat-band feature shown in the upper left insert of Fig. 1. It appears at the top of the $\sigma$ bands and extends with gradually fading intensity towards $\bar{K}$. Similar flat bands have been reported previously for graphene [30,31] and are attributed to the avoided band crossing of the back-folded bands. In the present case the flat band originates primarily from $\mathrm{N} p$ levels, since the top of the $\sigma$ band is dominated by $\mathrm{N} p$ orbitals oriented within the h-BN film plane.

The binding energies of the h-BN/Pt(110) bands compare well with those of a variety of h-BN/metal systems, if referred to the vacuum level. The work-function decrease $\Phi_{\mathrm{hBN} / \mathrm{Pt}}-\Phi_{\mathrm{Pt}}$ amounts to modest $760 \mathrm{meV}$. In contrast, for h-BN/Ni(111) $\Delta \Phi \approx-1.75 \mathrm{eV}$, signaling a much stronger interaction. Consequently, for the $\pi$ and $\sigma$ bands of $h-$ $\mathrm{BN} / \mathrm{Ni}(111)$, the vacuum level reference is apparently not entirely appropriate. If entered into Fig. 3, the binding energies for $\mathrm{h}-\mathrm{BN} / \mathrm{Ni}(111)$ would be consistently higher than average. Bokdam et al. [42] showed that for $\mathrm{Ni}, \mathrm{Co}$, and $\mathrm{Ti}$ the bonding interaction can be described as dominated by a covalent chemisorptive bond. By comparison, the present results indicate a weaker h-BN/Pt(110) interaction, which re- 
sults predominantly from nonlocal van der Waals bonding, but, according to the DFT results, still contains a considerable hybridization. The situation is somewhat reminiscent of how the precursor molecule borazine binds to $\mathrm{Pt}(110)$ [43]. This conclusion conforms to the theoretical analysis of Bokdam et al. [42]. Our previous results on the h-BN/Pt(110) geometry [17] indicated that the $\mathrm{N}$-metal distances lie between 2.3 and $3.3 \AA$, which according to Bokdam et al. is close to the transition between chemisorption and physisorption. Examination of the LDOS (see Fig. 4) reveals indeed a localized bonding contribution for $\mathrm{N}$ atoms located in on-top positions about 2.3 $\AA$ above $\mathrm{Pt}$ atoms. The related $\mathrm{Pt} d_{\mathrm{z}}-\mathrm{N} p_{\mathrm{z}}$ antibonding feature is pushed up to and beyond the Fermi level, giving rise to a small charge transfer from $\mathrm{N}$ to the metal. This is consistent with the lowering of the work function upon h-BN overlayer formation. The local bonding contribution stabilises the $(1 \times$ n) m.r. reconstruction of the Pt surface underneath the h-BN monolayer, which would be difficult to explain on the basis of a pure, nonlocal van der Waals bonding. Since the layer is grown significantly above the roughening temperature where the Pt atoms are very mobile, the m.r. reconstruction obviously forms during the cooling-down stage. After returning to room temperature the differences in thermal expansion result in many substrates in wrinkle formation. Here, it is the reconstruction of the substrate which accommodates the mismatch in [001] direction while in the orthogonal direction pinning is apparently no problem.

Finally, we address a remarkable result contained in Fig. 4: The LDOS within the h-BN band gap is negligible above the Pt missing rows, but contains considerable structure, where $\mathrm{N}$ atom positions coincide with the Pt close-packed rows. Accordingly, one may expect a considerable stripe-wise modulation of the chemical reactivity of the h-BN/Pt(110) system, which renders it an interesting candidate for cluster deposition and for catalysis studies.

\section{CONCLUSIONS}

$\mathrm{h}-\mathrm{BN} / \mathrm{Pt}(110)$ is a borderline case between nonlocal van der Waals and strong local interaction. On the one hand, the work-function change as well as the $\pi$ and $\sigma$ band binding energies, if referenced to the vacuum energy, conform with other weakly interacting h-BN/metal systems. The limited interaction strength gives rise to correlation effects showing up in both, a shift to higher binding energy of the ARPES final states and the resemblance of the ARPES bands to the free-standing monolayer. On the other hand, the structural corrugation, the calculated band structure, and the LDOS of $\mathrm{N}$ atoms adsorbed close to on-top positions provides clear evidence for a local covalent bonding. The contribution of this local bonding interaction is strong enough to induce after cooling-down a missing-row reconstruction with alternating periods of five or six Pt lattice constants. This is an exceptional case, where the substrate adapts to the adlayer rather than inducing stress or wrinkles. In ARPES the Moiré-induced modulation of the h-BN monolayer causes the appearance of replica bands shifted by the reciprocal-lattice vector $G_{1 \overline{1} 0}^{\text {Moire }}$ along the $k$ axis. The residual LDOS in the h-BN band gap varies considerably between on-top and above-missing-row positions. This should result in a similar local variability of the chemical reactivity.

\section{ACKNOWLEDGMENTS}

We thank the University of Innsbruck for financial support through the research platform "Advanced Materials" and the Doktoratskolleg "Reactivity and Catalysis." F.M. gratefully acknowledges the Austrian Science Fund (FWF, Project No. F45) for financial support and the Vienna Scientific Cluster for providing computing time.
[1] W. Auwärter, Hexagonal boron nitride monolayers on metal supports: Versatile templates for atoms, molecules and nanostructures, Surf. Sci. Rep. 74, 1 (2019).

[2] M. Corso, T. Greber, and J. Osterwalder, h-BN on Pd(110): a tunable system for self-assembled nanostructures? Surf. Sci. 577, L78 (2005).

[3] S. Berner, M. Corso, R. Widmer, O. Groening, R. Laskowski, P. Blaha, K. Schwarz, A. Goriachko, H. Over, S. Gsell, M. Schreck, H. Sachdev, T. Greber, and J. Osterwalder, Boron nitride nanomesh: functionality from a corrugated monolayer, Angew. Chem. Int. Ed. 46, 5115 (2007).

[4] M. Will, N. Atodiresei, V. Caciuc, P. Valerius, C. Herbig, and T. Michely, A monolayer of hexagonal boron nitride on $\operatorname{Ir}(111)$ as a template for cluster superlattices, ACS Nano 12, 6871 (2018).

[5] H. Jin, C. Guo, X. Liu, J. Liu, A. Vasileff, Y. Jiao, Y. Zheng, and S.-Z. Qiao, Emerging two-dimensional nanomaterials for electrocatalysis, Chem. Rev. 118, 6337 (2018).

[6] G. Elumalai, H. Noguchi, and K. Uosaki, Electrocatalytic activity of various types of h-BN for the oxygen reduction reaction, Phys. Chem. Chem. Phys. 16, 13755 (2014).
[7] H. L. Stern, R. Wang, Y. Fan, R. Mizuta, J. C. Stewart, L.-M Needham, T. D. Roberts, R. Wai, N. S. Ginsberg, D. Klenerman, S. Hofmann, and S. F. Lee, Spectrally resolved photodynamics of individual emitters in large-area monolayers of hexagonal boron nitride, ACS Nano 13, 4538 (2019).

[8] L. J. Martínez, T. Pelini, V. Waselowski, J. R. Maze, B. Gil, G. Cassabois, and V. Jacques, Efficient single photon emission from a high-purity hexagonal boron nitride crystal, Phys. Rev. B 94, 121405(R) (2016).

[9] D. Pierucci, J. Zribi, H. Henck, J. Chaste, M. G. Silly, F. Bertran, P. L. Fevre, B. Gil, A. Summerfield, P. H. Beton, S. V. Novikov, G. Cassabois, J. E. Rault, and A. Ouerghi, Van der Waals epitaxy of two-dimensional single-layer h-BN on graphite by molecular beam epitaxy: Electronic properties and band structure, Appl. Phys. Lett. 112, 253102 (2018).

[10] R. J. Koch, J. Katoch, S. Moser, D. Schwarz, R. K. Kawakami, A. Bostwick, E. Rotenberg, C. Jozwiak, and S. Ulstrup, Electronic structure of exfoliated and epitaxial hexagonal boron nitride, Phys. Rev. Mater. 2, 074006 (2018).

[11] A. Nagashima, N. Tejima, Y. Gamou, T. Kawai, and C. Oshima, Electronic states of monolayer hexagonal boron ni- 
tride formed on the metal surfaces, Surf. Sci. 357, 307 (1996).

[12] M. Morscher, M. Corso, T. Greber, and J. Osterwalder, Formation of single layer h-BN on Pd(111), Surf. Sci. 600, 3280 (2006).

[13] A. Goriachko, Y. B. He, and H. Over, Complex growth of NanoAu on BN nanomeshes supported by $\mathrm{Ru}(0001)$, J. Phys. Chem. C 112, 8147 (2008).

[14] S. Roth, T. Greber, and J. Osterwalder, Some like it flat: decoupled h-BN monolayer substrates for aligned graphene growth, ACS Nano 10, 11187 (2016)

[15] D. Usachov, A. Fedorov, O. Vilkov, V. K. Adamchuk, L. V. Yashina, L. Bondarenko, A. A. Saranin, A. Grüneis, and D. V. Vyalikh, Experimental and computational insight into the properties of the lattice-mismatched structures: Monolayers of h-BN and graphene on $\operatorname{Ir}(111)$, Phys. Rev. B 86, 155151 (2012).

[16] S. Achilli, E. Cavaliere, T. H. Nguyen, M. Cattelan, and S. Agnoli, Growth and electronic structure of 2D hexagonal nanosheets on a corrugated rectangular substrate, Nanotechnology 29, 485201 (2018).

[17] D. Steiner, F. Mittendorfer, and E. Bertel, Quasiliquid layer promotes hexagonal boron nitride (h-BN) single-domain growth: h-BN on Pt(110), ACS Nano 13, 7083 (2019).

[18] J. S. Lee, S. H. Choi, S. J. Yun, Y. I. Kim, S. Boandoh, J.-H. Park, B. G. Shin, H. Ko, S. H. Lee, Y.-M. Kim, Y. H. Lee, K. K. Kim, and S. M. Kim, Wafer-scale single-crystal hexagonal boron nitride film via self-collimated grain formation, Science 362, 817 (2018).

[19] See Supplemental Material at http://link.aps.org/supplemental/ 10.1103/PhysRevResearch.2.043156 for determination of the work function, LEED pattern, and dispersion of the h-BN band structure in other directions.

[20] D. Nečas and P. Klapetek, Gwyddion: an open-source software for SPM data analysis, Cent. Eur. J. Phys. 10, 181 (2012).

[21] G. Kresse and J. Furthmüller, Efficiency of ab-initio total energy calculations for metals and semiconductors using a plane-wave basis set, Comput. Mater. Sci. 6, 15 (1996).

[22] G. Kresse and J. Hafner, Ab initio molecular dynamics for liquid metals, Phys. Rev. B 47, 558 (1993).

[23] J. P. Perdew, K. Burke, and M. Ernzerhof, Generalized Gradient Approximation Made Simple, Phys. Rev. Lett. 77, 3865 (1996).

[24] S. Grimme, J. Antony, S. Ehrlich, and H. Krieg, A consistent and accurate $a b$ initio parametrization of density functional dispersion correction (DFT-D) for the 94 elements H-Pu, J. Chem. Phys. 132, 154104 (2010).

[25] F. Mittendorfer, A. Garhofer, J. Redinger, J. Klimeš, J. Harl, and G. Kresse, Graphene on Ni(111): Strong interaction and weak adsorption, Phys. Rev. B 84, 201401(R) (2011).

[26] P. V. C. Medeiros, S. Stafström, and J. Björk, Effects of extrinsic and intrinsic perturbations on the electronic structure of graphene: Retaining an effective primitive cell band structure by band unfolding, Phys. Rev. B 89, 041407(R) (2014).

[27] P. V. C. Medeiros, S. S. Tsirkin, S. Stafström, and J. Björk, Unfolding spinor wave functions and expectation values of general operators: Introducing the unfolding-density operator, Phys. Rev. B 91, 041116(R) (2015).

[28] X. Blase, A. Rubio, S. G. Louie, and M. L. Cohen, Quasiparticle band structure of bulk hexagonal boron nitride and related systems, Phys. Rev. B 51, 6868 (1995).
[29] P. Giraud, Study of the Electronic Structure of hexagonal Boron Nitride on Metals Substrates, Universit'e des Sciences et Technologies Lille 1 (Universidad del Pais Vasco - Euskal Herriko Unibertsitatea, San Sebastian, 2012).

[30] Y. Lin, G. Chen, J. T. Sadowski, Y. Li, S. A. Tenney, J. I. Dadap, M. S. Hybertsen, and R. M. Osgood, Observation of intercalation-driven zone folding in quasi-freestanding graphene energy bands, Phys. Rev. B 99, 035428 (2019).

[31] N. Ehlen, M. Hell, G. Marini, E. H. Hasdeo, R. Saito, Y. Falke, M. O. Goerbig, G. Di Santo, L. Petaccia, G. Profeta, and A. Grüneis, Origin of the flat band in heavily Cs-Doped graphene, ACS Nano 14, 1055 (2020).

[32] M. Minca, S. Penner, E. Dona, A. Menzel, E. Bertel, V. Brouet, and J. Redinger, Surface resonances on transition metals as lowdimensional model systems, New J. Phys. 9, 386 (2007)

[33] G. N. Derry, M. E. Kern, and E. H. Worth, Recommended values of clean metal surface work functions, J. Vac. Sci. Technol. A 33, 060801 (2015).

[34] D. Pacilé, P. Leicht, M. Papagno, P. M. Sheverdyaeva, P. Moras, C. Carbone, K. Krausert, L. Zielke, M. Fonin, Y. S. Dedkov, F. Mittendorfer, J. Doppler, A. Garhofer, and J. Redinger, Artificially lattice-mismatched graphene/metal interface: Graphene/Ni//r(111), Phys. Rev. B 87, 035420 (2013).

[35] F. Patthey, J. M. Imer, W. D. Schneider, H. Beck, Y. Baer, and B. Delley, High-resolution photoemission study of the lowenergy excitations in 4f-electron systems, Phys. Rev. B 42, 8864 (1990).

[36] V. Averbukh and L. S. Cederbaum, Ab initio calculation of interatomic decay rates by a combination of the Fano ansatz, Green's-function methods, and the Stieltjes imaging technique, J. Chem. Phys. 123, 204107 (2005).

[37] C. Keller, M. Stichler, G. Comelli, F. Esch, S. Lizzit, D. Menzel, and W. Wurth, Femtosecond dynamics of adsorbate charge-transfer processes as probed by high-resolution corelevel spectroscopy, Phys. Rev. B 57, 11951 (1998).

[38] J. C. Fuggle, Screening effects in core-level spectroscopies, in Inner-Shell and X-Ray Physics of Atoms and Solids. Physics of Atoms and Molecules, edited by D. J. Fabian, H. Kleinpoppen, and L. M. Watson (Springer, Boston, MA, 1981), pp. 485-488.

[39] T. Brugger, S. Günther, B. Wang, J. H. Dil, M.-L. Bocquet, J. Osterwalder, J. Wintterlin, and T. Greber, Comparison of electronic structure and template function of single-layer graphene and a hexagonal boron nitride nanomesh on $\mathrm{Ru}(0001)$, Phys. Rev. B 79, 045407 (2009).

[40] M. Corso, W. Auwärter, M. Muntwiler, A. Tamai, T. Greber, and J. Osterwalder, Boron nitride nanomesh, Science 303, 217 (2004).

[41] T. Greber, M. Corso, and J. Osterwalder, Fermi surfaces of single layer dielectrics on transition metals, Surf. Sci. 603, 1373 (2009).

[42] M. Bokdam, G. Brocks, M. I. Katsnelson, and P. J. Kelly, Schottky barriers at hexagonal boron nitride/metal interfaces: A first-principles study, Phys. Rev. B 90, 085415 (2014).

[43] L. Haug, J. P. Roth, M. Thaler, D. Steiner, A. Menzel, S. Tosoni, G. Pacchioni, and E. Bertel, Precursor chemistry of h-BN: adsorption, desorption, and decomposition of borazine on $\operatorname{Pt}(110)$, Phys. Chem. Chem. Phys. 22, 11704 (2020). 